\title{
MODELOS DE PREVISÃO DO TEOR DE UMIDADE ÓTIMO E DO PESO ESPECÍFICO SECO MÁXIMO PARA SOLOS FINOS
}

\author{
Models for estimating the optimum moisture and maximum \\ dry density for fine soils
}

\author{
Breno Padovezi Rocha ${ }^{1}$, Roberto Aguiar dos Santos ${ }^{2}$, Iraydes Tálita de Sena Nola ${ }^{3}$, \\ Flavio Alessandro Crispim ${ }^{4}$, André Luis Christoforo ${ }^{5}$
}

Recebido em 17 de novembro de 2017; aceito em 02 de abril de 2018; disponível on-line em 26 de maio de 2019.

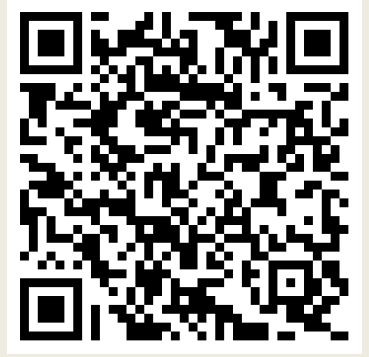

\section{PALAVRAS CHAVE:}

Ensaio de compactação; Modelos de regressão; Solos finos; Propriedades dos solos; Ensaios de laboratório.

\section{KEYWORDS:}

Compaction test; Regression models; Fine soils; Soil properties; Laboratory tests.
RESUMO: O dimensionamento de taludes, pavimentos rodoviários, liners, túneis, aterros entre outras aplicações civis, requer o conhecimento do comportamento dos solos através das suas propriedades físicas. Os solos podem apresentar comportamento distinto devido à variabilidade do maciço e ao processo de amostragem. O teor de umidade ótimo $\left(w_{\mathrm{ot}}\right)$ e o peso específico seco máximo $\left(\gamma_{\mathrm{dmax}}\right)$ são propriedades físicas fundamentais na análise do comportamento de um solo compactado e, podem ser influenciadas pela fração de pedregulho, fração de areia, fração de finos e pelos limites de liquidez e de plasticidade do solo. Para a viabilidade de projetos básicos pode ser necessário recorrer a formulações empíricas através das quais os limites de confiabilidade sejam conhecidos. Este trabalho objetivou investigar, com o auxílio da análise de variância de modelos de regressão, a precisão da estimativa do teor de umidade ótimo e do peso específico seco por meio dos ensaios corriqueiros de caracterização do solo. Assim, foram selecionados dados de 250 amostras de solos argilosos de diferentes regiões do planeta. Os resultados dos modelos de regressão multilinear dos solos argilosos investigados foram considerados expressivos pela ANOVA, apresentando valores do coeficiente de determinação ajustado para $w_{\text {ot }}$ e $\gamma_{\text {dmax }}$ iguais a 0,741 e 0,639, respectivamente. Com relação às propriedades físicas, apenas o limite de liquidez não foi significativo na estimativa do $\gamma_{\text {dmax }}$. As correlações empíricas apresentadas possibilitam a estimativa do teor de umidade ótimo e do peso específico seco máximo de maneira rápida e eficiente para a análise do comportamento dos solos compactados.

ABSTRACT: The design of slopes, road pavements, liners, tunnels, landfills among other civil applications requires the knowledge of the behavior of the soils through their physical properties. Soils may exhibit distinct behavior due to mass variability and the sampling process. The optimum moisture content $\left(w_{o t}\right)$ and maximum dry specific ( $\gamma_{\text {dmax. }}$ ) are fundamental physical properties in the analysis of compacted soil behavior and can be influenced by the fraction of gravel, sand fraction, fines fraction and limits liquidity and soil plasticity. For the feasibility of basic projects it may be necessary to resort to empirical formulations in which their reliability limits are known. The objective of this research was to investigate the accuracy of the estimation of the optimum moisture content and the maximum dry specific by means of the standard soil characterization tests with the aid of analysis of variance of regression models. Thus, data were selected from 250 samples of clayey soils from different regions of the word. The results of the multilinear regression models of the clayey soils investigated were considered expressive by ANOVA, presenting values of the coefficient of determination adjusted for wot and $\gamma_{\text {dmax }}$ equal to 0,741 and 0,639, respectively. Regarding the physical properties, only the liquidity limit was not significant in the estimation of $\gamma_{\text {dmax }}$. The proposed empirical relationships are able to give a reasonably good prediction on the optimum moisture content $\left(w_{o t}\right)$ and maximum dry specific $\left(\gamma_{\mathrm{dmax}}.\right)$ for evaluate the compacted soil behavior.

* Contato com os autores na página seguinte: 
* Contato com os autores:

${ }^{1}$ e-mail: breno_rochaa@hotmail.com (B. P. Rocha )

Engenheiro Civil, Doutor em Geotecnia pela Escola de Engenharia de São Carlos (EESC), da Universidade de São Paulo (USP).

2e-mail: robertoa.s@hotmail.com ( R. A. dos Santos )

Eng. Civil, MSc. Professor Assistente da Faculdade de Engenharia da Universidade Federal de Mato Grosso (UFMT), campus Várzea Grande/MT - Brasil.

${ }^{3}$ e-mail: talitanola@gmail.com (I.T.S. Nola )

Geóloga, MSc. Doutoranda em Geotecnia pela Universidade Federal de Ouro Preto, campus Morro do Cruzeiro, MG - Brasil.

${ }^{4}$ e-mail: crispim.br@gmail.com (F.A. Crispim )

Engenheiro Civil, Doutor em Engenharia Civil pela Universidade Federal de Viçosa (UFV). Professor do Departamento de Engenharia Civil da Universidade do Estado de Mato Grosso (UNEMAT), campus Sinop/MT - Brasil.

${ }^{4}$ e-mail: christoforoal@yahoo.com.br (A.L. Christoforo )

Eng. Civil, Doutor em Engenharia de Estruturas pela Escola de Engenharia de São Carlos (EESC), da Universidade de São Paulo (USP).

Professor do Departamento de Engenharia Civil da Universidade Federal de São Carlos (UFSCar), campus São Carlos/SP - Brasil.

\section{INTRODUÇÃO}

A compactação do solo é um processo de densificação que na maioria dos casos é realizada por meio de um equipamento mecânico, geralmente um rolo compactador, embora em alguns casos como em pequenas valetas, soquetes manuais possam ser empregados. $\mathrm{O}$ tipo de obra e o solo disponível na região do projeto vão ditar a maneira como o solo será compactado. $\mathrm{O}$ processo de compactação depende do teor de umidade, da densidade a ser atingida e do tipo de solo a ser compactado. A compactação do solo em uma obra tem como objetivo impedir futuros recalques, aumentar a rigidez do solo, e reduzir sua permeabilidade. Deste modo, fica clara a importância do controle de compactação no desempenho de obras geotécnicas.

A compactação é empregada em diversas obras de engenharia como em aterros, nas camadas constitutivas dos pavimentos, na construção de barragens de terra, entre outros (Sinha e Wang, 2008). Em campo, solos argilosos são compactados normalmente através de rolos do tipo pé de carneiro, rolos pneumáticos ou soquetes manuais. Em laboratório, a compactação do solo é realizada utilizando o equipamento do ensaio de Proctor. A compactação de solos finos depende do peso específico seco, teor de umidade, energia de compactação, tipo de solo e da sua granulometria (Santos, Rocha e Christoforo, 2015).
A partir do ensaio Proctor é possível determinar o peso específico seco máximo e o teor de umidade ótimo. No Brasil o ensaio é normatizado pela NBR 7182 (ABNT, 2016). Entretanto, a realização do ensaio de compactação em laboratório demanda tempo e esforços significativos (Nagaraj et al., 2015). Desse modo, para avaliação preliminar da adequação de um determinado solo para um projeto de interesse, pode-se recorrer a simples propriedades físicas, como os limites de consistência, por exemplo (Nagaraj et al., 2015). Os ensaios de limites de consistência são de simples determinação e bastante utilizados em correlações na engenharia geotécnica (Wesley, 2003).

Metodologias para a estimativa do teor de umidade ótimo e do peso específico seco máximo a partir de modelos empíricos têm sido desenvolvidas por diversos autores desde a década de 1940 (Rowan e Graham, 1948; Davidson e Gardiner, 1949; Sivrikaya, 2008). Normalmente, as equações desenvolvidas para a estimativa do teor de umidade ótimo e do peso específico seco máximo são baseadas no limite de liquidez, no limite de plasticidade, na densidade relativa dos grãos e a curva granulométrica. Rowam e Graham (1948) utilizaram a granulometria, a densidade relativa dos grãos e os limites de consistência na aplicação de suas equações de correlação. Davidson e Gardiner (1949) utilizaram o índice de plasticidade, a granulometria e a densidade 
relativa dos grãos. Turnbull (1948) correlacionou o teor de umidade ótimo e a granulometria e, Jumikis (1946) correlacionou teor de umidade ótimo e, limite de plasticidade e liquidez.

Ramia, Viswanath e Krishnamurthy (1970) correlacionaram teor de umidade ótimo e o peso específico máximo seco diretamente com o limite de liquidez. Jeng e Strohm (1976) correlacionaram o teor de umidade e o peso específico máximo seco com propriedades índice de 85 solos diferentes. Blotz, Benson, Boutwell (1998) utilizaram o teor de umidade ótimo e o peso específico seco obtidos a partir de 22 ensaios Proctor realizados em solos finos para obter correlações baseadas no limite de liquidez e na energia de compactação. Gurtug e Sridharan (2002 e 2004) correlacionaram o peso específico máximo seco e o teor de umidade ótimo de solos compactados de granulometria fina com o limite de plasticidade e a energia de compactação. Sridharan e Nagaraj (2005) correlacionaram o teor de umidade ótimo e o peso específico seco máximo com o limite de liquidez e de plasticidade em resultados de ensaios de na energia Proctor normal encontrados na literatura. Recentemente, diversos estudos têm utilizados modelos de regressão multilinear na estimativa do peso específico seco máximo e do teor de umidade ótimo (Sivrikaya, 2008; Gunaydin, 2008).

Por mais que existam diversos estudos baseados na estimativa das propriedades de compactação, poucos estudos procuram avaliar o nível de confiança desses modelos estatísticos gerados (Santos, Rocha, Christoforo, 2015). Esta avaliação é importante na escolha dos parâmetros e modelos que melhor explicam os fenômenos analisados, além disso, essas análises têm aplicação até mesmo na própria escolha dos modelos estatísticos mais apropriados.

A presente pesquisa tem como objetivo criar modelos que representem o comportamento dos solos finos através do auxílio da análise de variância de modelos de regressão, a precisão da estimativa do teor de umidade ótimo $\left(w_{o t}\right)$ e do peso específico seco $\left(\gamma_{\mathrm{dmax}}\right)$ como função das variações dos limites de liquidez $\left(w_{L}\right)$ e de plasticidade $\left(W_{P}\right)$ e das frações de pedregulho $(G)$, de areia (S) e de finos (FC) para solos categorizados finos pelo Sistema Unificado de Classificação dos Solos (SUCS).

\section{MATERIAL E MÉTODO}

Neste trabalho foram utilizados dados provenientes de resultados de ensaios realizados em 9 pesquisas distintas, explicitados na Tabela 1. Tais dados advêm dos ensaios de compactação Proctor Normal, propriedades índice (limite de liquidez, limite de plasticidade) e a distribuição granulométrica, na condição de dados pareados. 0 banco de dados utilizado é composto por 250 amostras e foi constituído pelas informações das amostras que apresentaram mais de $50 \%$ da fração de finos ( $F C$ ), ou seja, mais do que $50 \%$ passante na peneira de número 200. A Tabela 1 também apresenta as classes de solo categorizadas pela SUCS, por pesquisa, sendo $\mathrm{CH}$ (Argila de alta plasticidade), CL (argila de baixa plasticidade), ML (siltes de baixa plasticidade), MH (siltes de alta plasticidade).

\begin{tabular}{cccc}
\multicolumn{4}{c}{ TABELA 1: Resultados experimentais utilizados na presente pesquisa. } \\
Fonte & Classificação do solo & $\begin{array}{c}\text { No de resultados } \\
\text { utilizados }\end{array}$ & Localidade \\
\hline Sivrikaya (2008) & $\mathrm{CH} ; \mathrm{CL} ; \mathrm{ML}$ & 38 & Turquia \\
Berson e Trast (1995) & $\mathrm{CH} ; \mathrm{CL}$ & 11 & Estados Unidos \\
Gunaydin (2009) & $\mathrm{CL} ; \mathrm{ML}$ & 72 & Turquia \\
Horpibulsuk et al (2009) & $\mathrm{CH} ; \mathrm{CL}$ & 5 & Tailândia \\
Sinha e Wang (2008) & $\mathrm{CL}, \mathrm{ML} ; \mathrm{CH}-\mathrm{CL}$ & 5 & Estados Unidos \\
Yildirim e Gunaydin (2011) & $\mathrm{CH} ; \mathrm{CL} ; \mathrm{MH} ; \mathrm{ML}$ & 76 & Turquia \\
Mc Rae (1958) & $\mathrm{CH} ; \mathrm{CL} ; \mathrm{ML}$ & 12 & Estados Unidos \\
Alonso (2005) & $\mathrm{CL} ; \mathrm{MH} ; \mathrm{ML}$ & 4 & Brasil \\
Takeda (2006) & $\mathrm{MH} ; \mathrm{ML}$ & 27 & Brasil \\
\hline Total & & 250 & \\
\hline
\end{tabular}


Na Figura 1 estão plotados sobre a carta de plasticidade desenvolvida por Arthur Casagrande todos os dados amostrais. Nota-se que o banco de dados empregado é caracterizado por amostras que compreendem intervalos de limites de liquidez entre 20 e $80 \%$. Esse intervalo é compatível com a maioria dos solos disponíveis na literatura.

Sivrikaya (2008) utilizou 38 amostras de solo fino advindas de diferentes regiões da Turquia. Os dados provenientes desse autor apresentam elevada variabilidade na fração pedregulho e a fração areia. Berson e Trast (1995) utilizaram 11 amostras de solo fino empregado em liners de aterros sanitários nos Estados Unidos. Estas amostras apresentam grandes variações no limite de liquidez ( $\left.24 \% \leq w_{L} \leq 70 \%\right)$, no índice de plasticidade $(11 \% \leq \mathrm{IP} \leq 46 \%)$ e na fração de finos $(16 \% \leq \mathrm{FC} \leq 65 \%)$. Na pesquisa desenvolvida por Gunaydin (2009) foi utilizado redes neutrais artificiais na estimativa dos parâmetros de compactação. Das 126 amostras de solo disponíveis deste autor, apenas 73 amostras foram classificadas como solos finos. Horpibulsuk et al. (2009) propuseram um método simplificado para estimativa da curva de compactação em solos granulares e finos. Sinha e Wang (2008) propuseram modelos de previsão dos parâmetros de compactação e de permeabilidade a partir de redes neurais artificiais para solos compactados. Yildirim e Gunaydin (2011) desenvolveram modelos matemáticos para a previsão do CBR (California Bearing Ratio) por meio de propriedades índice e parâmetros de compactação. Tais modelos englobaram 76 amostras de solos finos coletadas em diversas rodovias públicas na Turquia. Mc Rae (1958) avaliou, através dos parâmetros de compactação, a energia de compactação necessária para alcançar o peso específico de campo desejado. Este estudo utilizou 17 amostras de solos finos.

Alonso (2005) avaliou a condutividade hidráulica de solos compactados com permeâmetro de parede flexível. $\mathrm{O}$ autor compactou 4 amostras de solos advindas de quatros regiões distintas do Estado de São Paulo. Takeda (2006) estudou o comportamento mecânico de solos rodoviários em função da variação da umidade pós-compactação. Neste estudo, o autor utilizou 72 amostras de solos finos advindas de diversos lugares no Estado de São Paulo. Convém destacar que a maioria dos dados obtidos por Takeda (2006) são caracterizados por solos de comportamento laterítico. A incorporação desses dados atribui uma maior representatividade aos modelos gerados, principalmente para aplicação em regiões de climas tropicais como o Brasil.

A partir dessas pesquisas foi possível construir um banco de dados e elaborar os modelos de previsão de teor de umidade ótimo e peso específico seco máximo para os solos desta

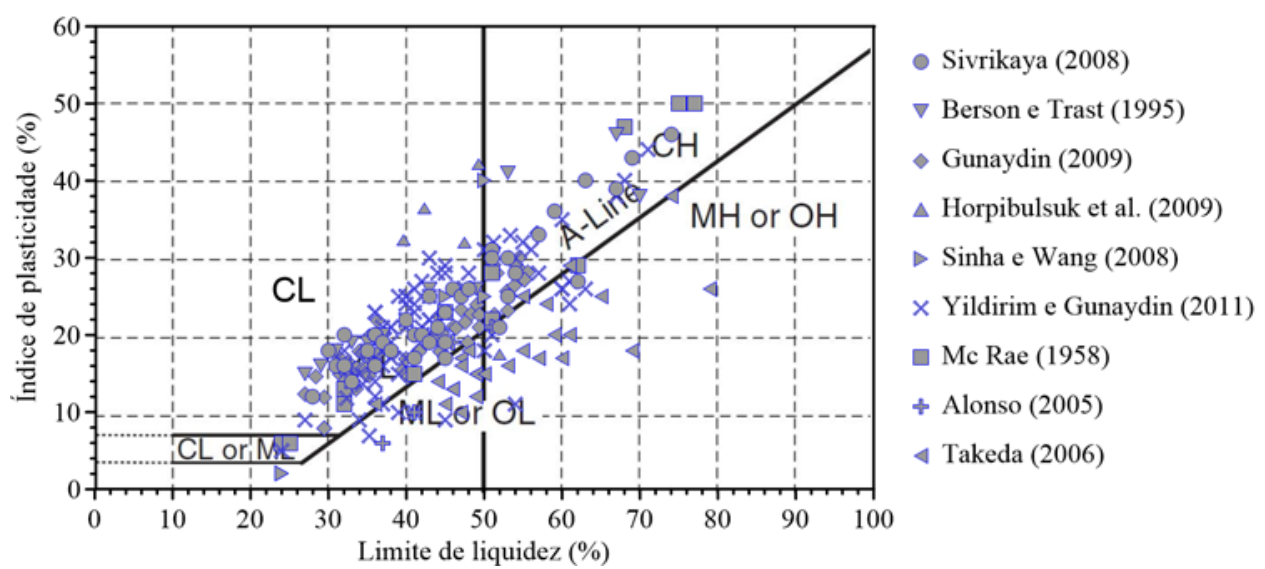

FIGURA 1: Carta de plasticidade de Casagrande e resultados experimentais utilizados na presente pesquisa. FONTE: Autoria Própria. 
categoria. Para verificar a significância das propriedades físicas utilizadas na estimativa do teor de umidade ótimo e do peso específico máximo seco dos solos foi realizada a análise de variância (ANOVA) dos modelos multilineares ajustados. A hipótese nula $\left(\mathrm{H}_{0}\right)$ foi considerada como a não significância da regressão pelos coeficientes ajustados e, a significância da regressão foi considerada como hipótese alternativa $\left(\mathrm{H}_{1}\right)$, ao nível de significância de $5 \%$. Pela formulação das hipóteses, P-valor superior a $5 \%(0,05)$ implica em aceitar $\mathrm{H}_{0}$, refutando-a em caso contrário.

\section{RESULTADOS E DISCUSSÕES}

\subsection{MODELO PARA ESTIMATIVA DO TEOR DE UMIDADE ÓTIMO}

A Tabela 2 apresenta os resultados da análise de variância dos modelos de regressão multilineares ajustados para os valores do teor de umidade ótimo $\left(w_{o t}\right)$ juntamente com o respectivo coeficiente de determinação ajustado ( $R^{2}[\operatorname{adj}]$ ). Observa-se nessa tabela que todos os P-valores foram considerados significativos pela ANOVA (P-valor $<0,05)$.

\section{TABELA 2: Teor de umidade ótimo em função das} demais propriedades físicas.

Modelo de Regressão: Equação 1

\begin{tabular}{cc}
\multicolumn{2}{c}{$\mathrm{R}^{2}[\mathrm{adj}]=0,741$} \\
\hline ANOVA & $P$-valor \\
\hline$c^{t e}$ & 0,002 \\
\hline Regressão & 0,000 \\
\hline$G$ & 0,006 \\
\hline$S$ & 0,002 \\
\hline$F C$ & 0,018 \\
\hline$w_{L}$ & 0,007 \\
\hline$w_{P}$ & 0,000
\end{tabular}

FONTE: Autoria Própria.

Pode-se observar um modelo de regressão multilinear de $w_{o t}$ significativo visto o valor de $\mathrm{R}^{2}$ [adj] de 0,741, sendo todas as propriedades investigadas expressivas na obtenção do $w_{o t}$. 0 valor de $R^{2}$ [adj] nesta pesquisa é condizente com o obtido por Gunaydin (2009). Na Equação 1 são apresentados os coeficientes ajustados às variáveis independentes:

$$
\begin{aligned}
& w_{o t}=67,06-0,60 \times G-0,65 \times S- \\
& \quad 0,50 \times F C-0,01 \times w_{L}+0,29 \times w_{p}
\end{aligned}
$$

\section{Em que:}

$\mathbf{w}_{\text {ot }}=$ Teor de umidade ótimo (\%);

$\mathbf{G}=$ Fração pedregulho (\%);

$\mathbf{S}=$ Fração areia (\%);

FC = Fração de finos (\%);

$\mathbf{W}_{\mathrm{L}}=$ Limite de liquidez (\%);

$\mathbf{W}_{\mathbf{p}}=$ Limite de plasticidade (\%).

Para verificar a aplicabilidade de modelos com menor quantidade de variáveis. A Equações 2, 3 e 4 apresentam valores de $R^{2}$ [adj] iguais a 0,557, $0,445,0,432$, respectivamente. Nestas equações, os modelos de regressão foram obtidos a partir da retirada progressiva das variáveis dependentes do modelo geral apresentado na Tabela 2, objetivando verificar o quanto cada variável influencia na estimativa do teor de umidade ótimo.

$$
\begin{aligned}
& \mathcal{W}_{o t}=93,38-0,84 \times G-0,88 \times S+ \\
& 0,70 \times F C+0,03 \times w_{L} \\
& W_{o t}=101,31-0,90 \times G-0,96 \times \\
& S-0,75 \times F C \\
& w_{o t}=25,56-0,14 \times G-0,21 \times S
\end{aligned}
$$

Nota-se que a retirada do limite de plasticidade proporcionou a maior redução (25\% aproximadamente) no valor do coeficiente de determinação ajustado ( $R^{2}$ [adj]). É possível perceber que o aumento do porcentual de areia (S) contribui na redução no valor estimado de teor de umidade ótimo, comportamento que, por sua vez, é o esperado para solos compactados. Em solos finos, o limite de liquidez e o limite de plasticidade explicam melhor a variabilidade do teor de umidade ótimo (Sivrikaya, Ergun e Cafer, 2008). A Equação 5 e 6 apresentam os modelos gerados levando em consideração apenas o limite de plasticidade e o limite de liquidez, respectivamente. Os modelos ajustados presentes na Equação 5 e 6 apresentam valores de coeficiente de determinação ajustado ( $R^{2}$ [adj]) 
iguais a 0,520 e 0,542, respectivamente. A Figura 2 mostra um comparativo entre os modelos gerados por Sivrikaya, Ergun e Cafer (2008) acompanhados dos modelos gerados na presente pesquisa (Equações 5 e 6).

$$
\begin{gathered}
w_{o t}=0,40 \times w_{P}+10,76 \\
w_{o t}=0,27 \times w_{L}+7,88
\end{gathered}
$$

Em que:

$$
\begin{aligned}
& \mathbf{w}_{\mathrm{ot}}=\text { Teor de umidade ótimo (\%); } \\
& \mathbf{w}_{\mathrm{L}}=\text { Limite de liquidez (\%); } \\
& \mathbf{w}_{\mathrm{P}}=\text { Limite de plasticidade (\%). }
\end{aligned}
$$

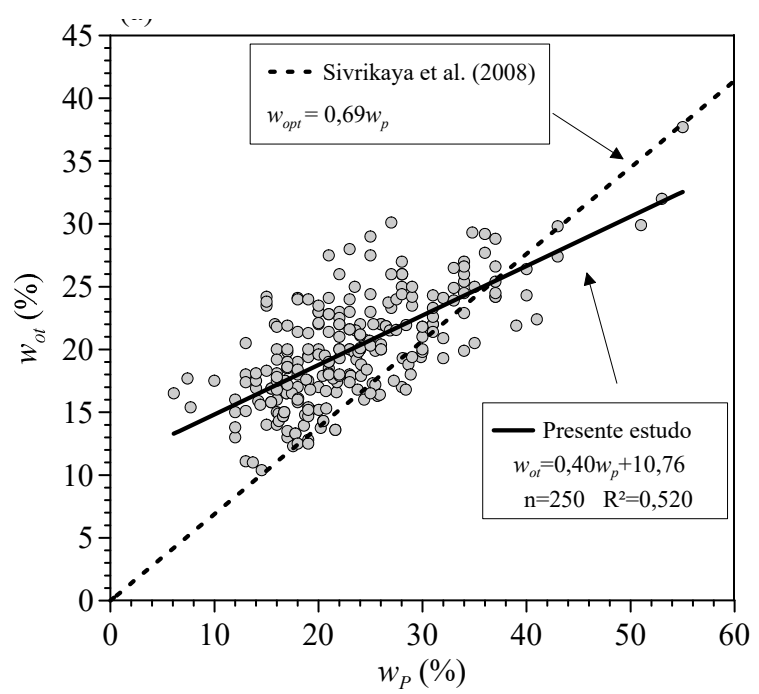

[a]

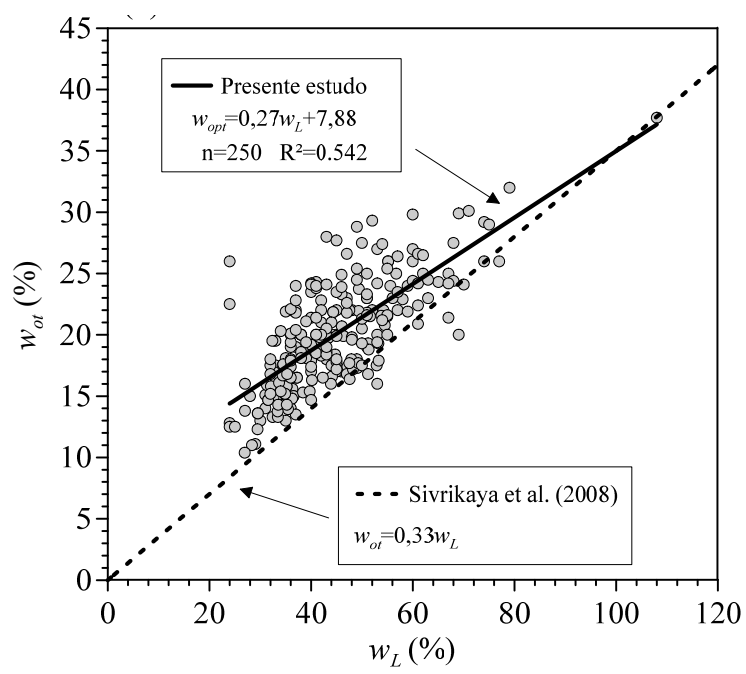

[b]

FIGURA 2: Comparação entre os modelos propostos na presente pesquisa e os propostos por Sivrikaya, Ergun e Cafer (2008). FONTE: Autoria Própria.
Observa-se nas Equações 5 e 6 que as equações desenvolvidas apresentam menor inclinação quando comparados aos modelos propostos por Sivrikaya, Ergun e Cafer (2008). Sendo assim, a Equação 1 consegue melhor explicar a variação do teor de umidade ótimo de solos compactados.

\subsection{MODELO PARA ESTIMATIVA DO PESO ESPECÍFICO SECO MÁXIMO}

Assim como foi feito com $w_{o t}$, também foram avaliados e propostos modelos de regressão na tentativa de explicar a variabilidade $g_{d m a x}$ para solos finos. A Tabela 3 apresenta os resultados da análise de variância do modelo de regressão multilinear ajustado para os valores peso específico seco máximo $\left(g_{d \max }\right)$ juntamente com o

\begin{tabular}{|c|c|}
\hline \multicolumn{2}{|c|}{$\begin{array}{c}\text { Modelo de Regressão: Equação } 7 \\
R^{2}[\operatorname{adj}]=0,639\end{array}$} \\
\hline ANOVA & $P$-valor \\
\hline$c^{t e}$ & $0,808^{\mathrm{ns}}$ \\
\hline Regressão & 0,000 \\
\hline$G$ & 0,007 \\
\hline$S$ & 0,011 \\
\hline$F C$ & 0,047 \\
\hline$w_{L}$ & $0,060^{\mathrm{ns}}$ \\
\hline$w_{P}$ & 0,000 \\
\hline
\end{tabular}
respectivo coeficiente de determinação ajustado ( $R^{2}[$ adj]). Além disso, a tabela também identifica os $\mathrm{P}$-valores considerados não significativos (ns) pela ANOVA (P-valor $>0.05$ ).

LEGENDA: ${ }^{\text {ns }}$ - não significativo FONTE: Autoria Própria.

Através da Tabela 3, observa-se que o modelo de regressão multilinear para a estimativa de $\gamma_{\text {dmax }}$ é significativo, apresentando valor de $\mathrm{R}^{2}$ [adj] de 0,639, e que dentre as variáveis independentes investigadas apenas o limite de liquidez $\left(w_{L}\right)$ não foi significativo. Isso implica que a variabilidade do peso específico seco pouco consegue ser explicada pelo limite de liquidez do solo. A Equação 7 apresenta a estimativa do peso específico com a retirada do limite de liquidez do modelo de regressão geral apresentado na Tabela 3. 


$$
\begin{aligned}
\gamma_{d \max }= & 2,03-0,20 \times G-0,19 \times S- \\
& 0,14 \times F C-0,08 \times w_{p}
\end{aligned}
$$

optou-se por avaliar a dispersão de $w_{L}$ junto aos pontos coletados.

$$
\begin{aligned}
& \gamma_{d \max }=18,66-0,100 \times w_{P} \\
& \gamma_{d \max }=19,68-0,077 \times w_{L}
\end{aligned}
$$

\section{Em que:}

$$
\begin{aligned}
& \gamma_{\mathrm{dmax}}=\text { Peso específico seco máximo }\left(\mathrm{kN} / \mathrm{m}^{3}\right) \\
& \mathbf{W}_{\mathrm{L}}=\text { Limite de liquidez }(\%) \\
& \mathbf{W}_{\mathbf{P}}=\text { Limite de plasticidade (\%). }
\end{aligned}
$$

de $\gamma_{\text {dmax }}$ das amostras utilizadas na presente pesquisa. Sridharan e Nagaraj (2005) comentam que utilizando $n=64$ foi possível obter uma ótima relação entre o peso específico seco e o limite de plasticidade das amostras. Na Figura 3 são apresentados os modelos que levam em consideração $0 \quad w_{P}$ e $\quad w_{L}$ como variáveis independentes, Equações 8 e 9 respectivamente, acompanhado dos modelos desenvolvidos por Sivrikay (2008). Mesmo sabendo que o teste pela ANOVA, apresentado na Tabela 3, indicou que o $w_{L}$ não foi significativo ao modelo da Equação 7 ,
A Figura 3 mostra que para valores de $\mathrm{W}_{\mathrm{p}}>35 \%$ a dispersão aumenta significativamente, - que pode ter contribuído para os baixos valores de $\mathrm{R}^{2}$ [adj]. Para valores de $w_{P}<35 \%$ o modelo proposto por Sivrikaya (2008) parece se ajustar bem aos pontos utilizados nesta pesquisa. Com relação ao limite de liquidez é possível perceber que ele consegue explicar bem a variação de $\gamma_{\mathrm{dmax}}$ (Figura $3 b$ ), conforme verificado também pelo teste estatístico apresentado na Tabela 3.
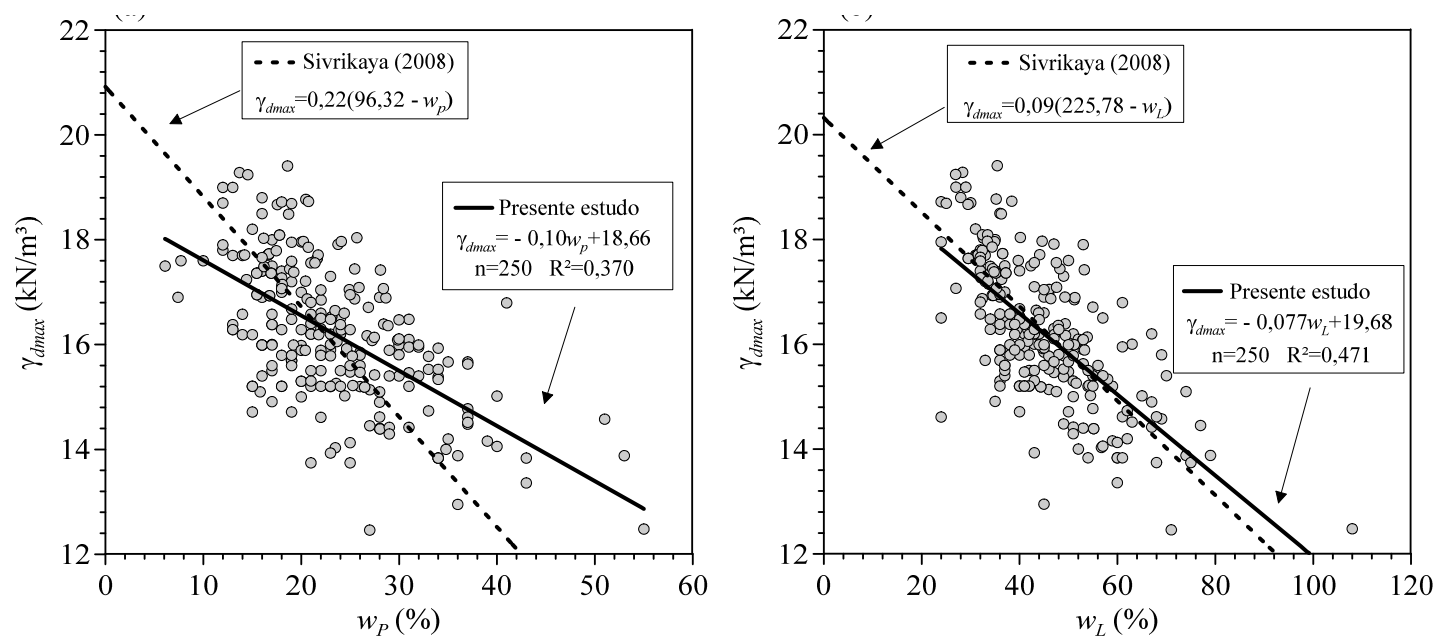

FIGURA 3: Comparação entre os modelos propostos na presente pesquisa e os propostos por Sivrikaya (2008).

FONTE: Autoria Própria. 


\subsection{MODELO PARA ESTIMATIVA DO PESO ESPECÍFICO SECO MÁXIMO COM O TEOR DE UMIDADE ÓTIMO}

Blot, Benson e Boutwell (1998), Sivrikaya

(2008), Sivrikaya, Ergun e Cafer (2008) apresentaram modelos empíricos para estimativa de $\gamma_{d \max }$ por meio da variação de $\left(w_{o t}\right)$ para solos de granulação fina. Neste trabalho foram gerados modelos de regressão (linear, quadrático, exponencial e logarítmico) para a estimativa do peso específico seco $\left(\gamma_{\text {dmax }}\right)$ em função do teor de umidade ótimo $\left(w_{o t}\right)$ dos solos. Acompanham as Equações 10 (modelo linear), 11 (modelo quadrático), 12 (modelo exponencial) e 13 (modelo logarítmico) com valores de $\mathrm{R}^{2}$ [adj] iguais a $0,803,0,813,0,814,0,812$, respectivamente. A Equação 12 (modelo exponencial) foi aquele que apresentou o melhor ajuste, no entanto, sempre é preferível ajustes lineares (Equação 10), mas que não difere significativamente dos demais ajustes aqui apresentados.

$$
\begin{array}{ccc} 
& \gamma_{d \text { max }}=21,62-0,26 \times w_{o t} & \text { Eq. [10] } \\
\gamma_{d \text { max }}= & 23,62-0,46 \times w_{o t}+0,004 \times w_{o t}{ }^{2} & \text { Eq. [11] } \\
& \gamma_{d \max }=22,69 \times e^{-0,01 \times w_{o t}} & \text { Eq. [12] } \\
& \gamma_{d \max }=32,48-5,46 \times \ln w_{o t} & \text { Eq. [13] }
\end{array}
$$

\section{Em que:}

$\gamma_{\mathrm{dmax}}=$ Peso específico seco máximo $\left(\mathrm{kN} / \mathrm{m}^{3}\right)$; $\mathbf{w}_{\text {ot }}=$ Teor de umidade ótimo (\%).

Para que fosse analisada a dispersão dos dados frente aos modelos gerados (Equações 10, 11,12 e 13), optou-se por sobrepô-los às famílias de curvas de compactação proposta por Joslin (1958). As curvas famílias de curvas desenvolvidas por Joslin (1958) são um conjunto de curvas de solo dos mais variados tipos, desenvolvidos originalmente nos anos de 1930 e 1940 que procura representar mais de 10.000 solos encontrados no estado de Ohio no Estados Unidos (EUA). Estas curvas foram obtidas utilizando energia Proctor Normal e são apresentadas na Figura 4. Além dos modelos propostos, também se encontra na Figura 4 o modelo desenvolvido por Sivrikaya (2008) para uma gama de 156 amostras. O modelo apresentado por Sivrikaya (2008) parece se ajustar bem aos pontos analisados e fica praticamente paralelo ao modelo linear apresentado na presente pesquisa (Equação 10).

Recentemente Santos, Rocha e Christoforo (2015) desenvolveram modelos empíricos para prever o comportamento de solos granulares compactados ( $\mathrm{FC}<50 \%$ ) e obtiveram um $R^{2}$ [adj] = 0,699 a partir de 133 amostras analisadas. Os modelos propostos pelos referidos autores também foram inseridos na Figura 4. Nota-se que para uma faixa de menores valores de teor de umidade $\left(12 \% \leq \mathrm{w}_{\text {ot }} \leq 24 \%\right)$ o modelo apresentado por Santos, Rocha e Christoforo (2015) se aproxima do modelo linear apresentado na presente pesquisa (Equação 10). Em outras palavras, por mais que o modelo seja desenvolvido para solos granulares, o mesmo consegue explicar também uma faixa de solos finos. A respeito do comportamento dos modelos desenvolvidos, a Equação 13 chama atenção para a boa concordância com os pontos de máximo $\left(\gamma_{\mathrm{dmax}} \mathrm{e}\right.$ $\mathrm{w}_{\mathrm{ot}}$ ) apresentados por Joslin (1958), inclusive para a região com menores teores de umidade.

Vale ressaltar que alguns pontos se encontram distantes das famílias de curvas apresentadas por Joslin (1958). Este comportamento pode estar associado às incertezas do ensaio, formação dos solos, mineralogia do solo, entre outros. Takeda (2006) é responsável por $10 \%$ do banco de dados utilizados neste trabalho. $O$ referido autor utilizou em seu trabalho amostras obtidas em solos residuais do interior do estado de São Paulo, onde devido à atuação do intemperismo podem apresentar significativa variação mineralógica ao longo do perfil. Fookes (1997) e Blight (1997) discutem a dificuldade do controle de qualidade na compactação em solos residuais, onde os procedimentos de preparação das amostras e de ensaios de laboratório podem ser significativos. 


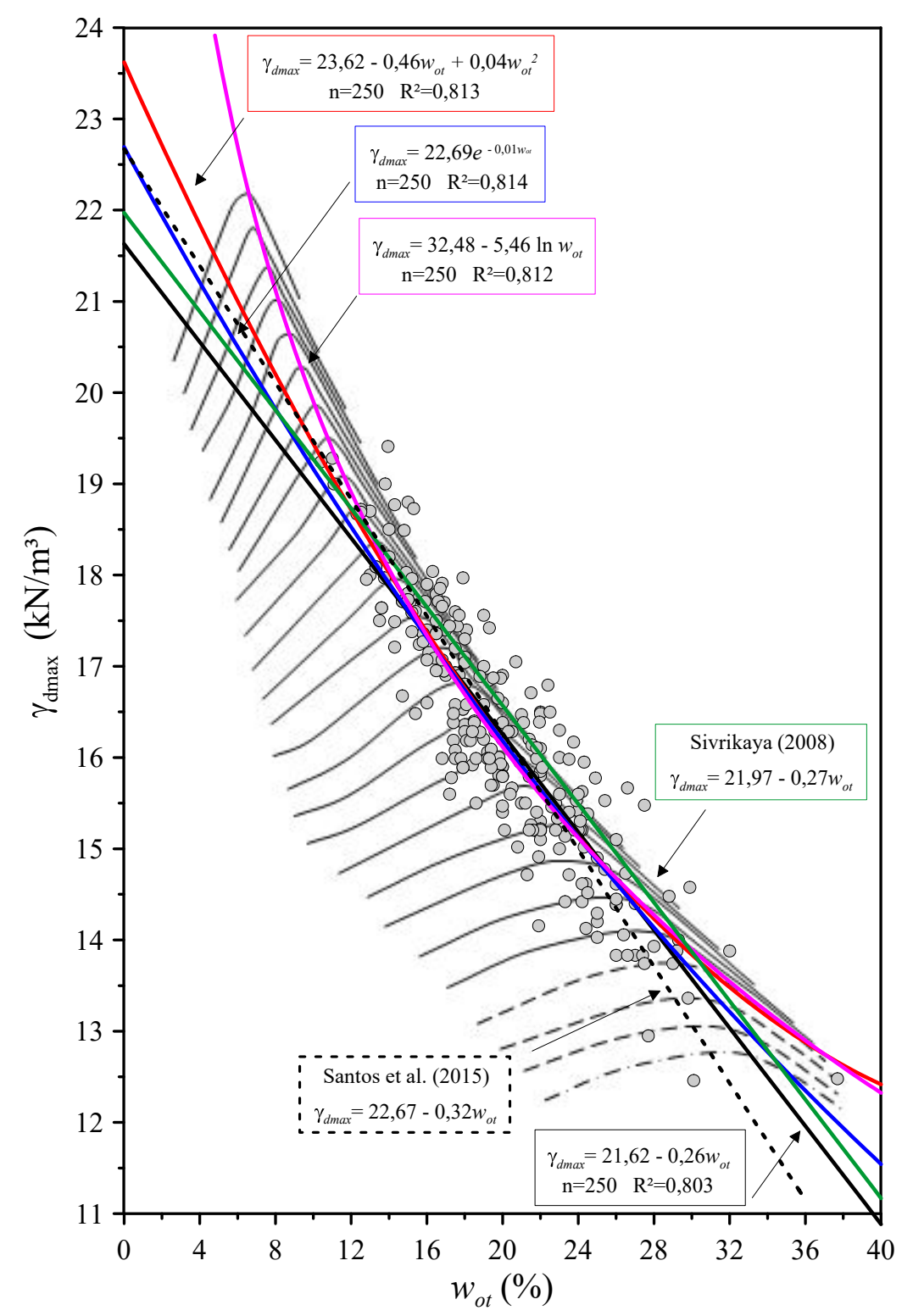

FIGURA 4: Dados utilizados na presente pesquisa e modelos elaborados na presente pesquisa. FONTE: Adaptado de Joslin (1958).

Estas incertezas tendem aumentar à medida que os solos apresentam elevado teor de umidade ótimo e elevados limites de liquidez, devido à dificuldade na obtenção dos parâmetros ótimos da curva de compactação $\left(\gamma_{\mathrm{dmax}}\right.$ e $\left.\mathrm{w}_{\mathrm{ot}}\right)$. Para solos com elevada plasticidade, onde o formato da curva de compactação é bastante achatado, o que pode contribuir com as incertezas na obtenção dos parâmetros ótimos da curva. Outro fato que influencia na compactação dos solos residuais é a presença de argilominerais metaestáveis, teor de umidade in situ, estrutura ligeiramente cimentada, além de sesquióxidos que são afetados por umedecimento e secagem (Takeda, 2006).

\section{CONCLUSÕES}

A compactação é empregada em diversas obras de engenharia, como os aterros, pavimentos rodoviários, barragens de terra. Assim, o desenvolvimento de modelos de regressão para a estimativa dos parâmetros de compactação, umidade ótima $\left(w_{o t}\right)$ e peso específico máximo seco $\left(\gamma_{\text {dmax }}\right)$ é de suma importância na fase preliminar à realização do projeto, seja por limitações financeiras ou até mesmo de tempo para execução preliminar do ensaio. 0 presente estudo organizou um banco de dados de solos disponíveis na literatura e propôs modelos 
de regressão dos parâmetros de compactação e dos índices físicos dos solos de diversas regiões do mundo.

Os resultados dos modelos de regressão linear múltipla para a estimativa do teor de umidade ótimo $\left(w_{o t}\right)$ e do peso específico seco $\left(\gamma_{\mathrm{dmax}}\right)$ dos solos argilosos aqui investigados foram considerados significativos pela ANOVA, apresentando valores do coeficiente de determinação ajustado iguais a 0,741 e 0,639, respectivamente.

Das propriedades físicas utilizadas na estimativa de $w_{\text {ot }}$ e $\gamma_{\text {dmax }}$, com exceção do limite de liquidez para o peso específico seco, as demais variáveis afetaram de forma significativa os valores das duas propriedades estimadas. Os modelos ajustados entre os limites de plasticidade e de liquidez mostraram os menores valores de $R^{2}$ [adj] na estimativa de $w_{\text {ot }}$ e $\gamma_{\text {dmax }}$, diferente do observado por Sivrikaya (2008). Este comportamento se deve principalmente a presença de solos com elevada plasticidade $w_{p}>$ $50 \%$ no banco de dados. Para solos nesta categoria o desenvolvimento de modelos fica comprometido, visto a elevada variabilidade dos parâmetros de compactação e dos limites de consistência.

No entanto, os resultados indicaram boa correlação entre $\gamma_{\mathrm{dmax}}$ e $\mathrm{W}_{\mathrm{ot}}$. A sobreposição dos modelos às famílias de curvas desenvolvidas por Joslin (1958) forneceu um indicativo da abrangência do banco de dados utilizados nesta pesquisa. Além disso, foi possível estabelecer regiões em os modelos analisados se aproximam entre si. A partir disso, pode-se destacar que estas correlações são úteis para aplicações práticas.

Mesmo tendo sido utilizado um conjunto com 250 amostras para cada uma das propriedades físicas de interesse, os resultados dos coeficientes de determinação ajustados dos modelos de regressão para o peso específico seco dos solos foram inferiores aos valores apresentados na literatura correlata. Todavia, a melhoria na precisão dos modelos pode ser atingida com o uso de um maior banco de dados e/ou com a estimativa destas propriedades estratificadas em função da classificação dos solos argilosos, o que motiva o desenvolvimento de outras pesquisas nesta temática. As correlações empíricas apresentadas possibilitaram a estimativa do teor de umidade ótimo e do peso específico seco máximo de maneira rápida e eficiente para a análise do comportamento dos solos compactados.

\section{REFERÊNCIAS BIBLIOGRÁFICAS}

ALONSO, T. Condutividade hidráulica de solos compactados de parede flexível. Tese (Mestrado Geotecnia) - Escola de Engenharia de São Carlos. Universidade de São Paulo. São Carlos, SP, 2005, 113 p.

ASSOCIAÇÃO BRASILEIRA DE NORMAS TÉCNICAS, NBR 7182: Solo - Ensaio de Compactação. Rio de Janeiro, 2016.

ASTM D 4318-95a,. Standard Test Methods for Liquid Limit, Plastic Limit, and Plasticity Index of Soil. Annual Book of ASTM Standards, Vol. 4, No. 8, ASTM International, West Conshohocken, PA. pp. 519-529, 1997.

ASTM D 698-91,. Test Methods for Laboratory Compaction Characteristics of Soil Using Standard Effort. Annual Book of ASTM Standards, Vol. 4, No. 8, ASTM International, West Conshohocken, PA. pp. 7784, 1997.

BENSON, C.H.; TRAST, J.M. Hydraulic conductivity of thirteen compacted clays. Clays and Clay Minerals, 1995, Vol. 43, n. 6, 669-681 p.

BLIGHT, G. E. Origin and formation of residual soils. In: BLIGHT, G. E. Mechanical of Residual Soils. Rotterdam, Brookfield: Ed. Balkema, 1997.

BLOTZ, L. R.; BENSON, C. H.; BOUTWELL, G. P. Estimating optimum water content and maximum dry unit weight for compacted clays. Journal of Geotechnical and Geoenvironmental Engineering, 1998, Vol. 124, n. 9, 907-912 p.

DAVIDSON, D. T.; GARDINER, W. F. Calculation of standard proctor density and optimum moisture content from mechanical, analysis, shrinkage and factors and plasticity index. Highway Research Board, Vol. 29, 1949, 447-481 p.

FOOKES, P. G., ed. Tropical residual soils: A Geological Society Engineering Group working party revised report. Geological Society of London, 1997.

GUNAYDIN, O. Estimation of compaction parameters by using statistical analyses and artificial neural networks. Environmental Geology Journal, 2009, Vol. 57, 203-215 p. 
GÜNAYDIN, O. Estimation of soil compaction parameters by using statistical analyses and artificial neural networks. Environmental Geology, Vol. 57, n. 1, 2009, 203.

GURTUG, Y.; SRIDHARAN, A. Prediction of compaction characteristics of fine-grained soils. Geotechnique, 2002, Vol. 52, n. 10, 761-763 p.

GURTUG, Y; SRIDHARAN, A. Compaction behavior and prediction of its characteristics of fine-grained soils with particular references to compaction energy. Soil Foundations, 2004, Vol. 44, n. 5, 27-36 p.

HORPIBULSUK, S.; KATKAN, W.; NARAMITKORNBUREE, A. Modified Ohio's Curves: A Rapid Estimation of Compaction Curves for Coarse- and Fine-Grained Soils. Geotechnical Testing Journal, 2009, Vol. 32, n.1, 64-75 p.

JENG, Y. S.; STROHM, W. E. Prediction of the shear strength and compaction characteristics of compacted fine-grained cohesive soils. United States Waterways Experiment Station, Soil and Pavement Laboratory, 1976, Vicksburg, Miss

JOSLIN, J. C. Ohio's typical moisture-density curves. American Society of Testing and Materials, 1958, ASTM STP, 239: 111-118.

JUMIKIS, A. R. Geology and Soils of the Newark (N.J.) Metropolitan Area, Journal of the Soil Mechanics and Foundations Division, ASCE, 1946, No. SM2.

Mc RAE, J. L. Index of compaction characteristics. American Society of Testing and Materials, 1958, ASTM STP 239: 119-27.

NAGARAJ, H. B.; REESHA, B.; SRAVAN, M. V.; SURESH, $M$. R. Correlation of compaction characteristics of natural soils with modified plastic limit. Transportation Geotechnics, Vol. 2, 2015, 65-77 p.

RAMIAH, B. K.; VISWANATH, V.; KRISHNAMURTHY, H. V. Interrelationship of Compaction and Index Properties, In: Second Southeast Asian Conference on Soil Engineering, Singapore, 1970, 577-587 p.

ROWAN, H. W.; GRAHAM, W. W. Proper Compaction Eliminates Curing Period in Construction Fills. Civil Engineering, 1948, Vol. 18, 450-451 p.

SANTOS, R. A.; ROCHA, B. P.; CHRISTOFORO, A. L. Estimativa da umidade ótima e do peso específico seco máximo para solos granulares compactados. In: 15th Pan-American Conference on Soil Mechanics and Geotechnical Engineering, 2015, Buenos Aires. 15th Pan-American Conference on Soil Mechanics and Geotechnical Engineering. Amsterdam: IOS Press BV, 2015. v. 1. p. 228-235.
SINHA, S. K.; WANG, M. C. Artificial neural network prediction models for soil compaction and permeability. Geotechnical and Geological Engineering, Vol. 26, n. 1, 2008, 47-64 p.

SIVRIKAYA, O. Models of compacted fine-grained soils used as mineral liner for solid waste. Environmental geology, Vol. 53, n. 7, 2008, 1585.

SIVRIKAYA, O; ERGUN T.; CAFER K. Estimating compaction behavior of fine-grained soils based on compaction energy. Canadian Geotechnical Journal, 2008, Vol. 45, n. 6, 877-887 p.

SRIDHARAN, A.; NAGARAJ, H. B. Plastic limit and compaction characteristics of fine-grained soils. Proceedings of the Institution of Civil Engineers-Ground Improvement, 2005, Vol. 9, n. 1, 17-22 p.

TAKEDA, M. C. influência da variação da umidade póscompactação no comportamento Mecânico de solos de rodovias do Interior paulista. Tese (Doutorado em Engenharia de Transportes) - Escola de Engenharia de São Carlos. Universidade de São Paulo. São Carlos, SP, 2006, 276 p.

TURNBULL, J. M. Computation of the Optimum Moisture Content in the Moisture-Density Relationship of Soils, In: Second International Conference on Soil Mechanics and Foundation Engineering, Rotterdam, Holland, Vol. IV, 1948, pp. 256262.

WESLEY, L. D. Residual strength of clays and correlations using Atterberg limits. Geotechnique, Vol. 53, n. 7, 2003, 669-672 p.

YILDIRIM, B.; GUNAYDIN, O. Estimation of California bearing ratio by using soft computing systems. Expert systems with applications, 2009, v. 38, n. 5, 6381-6391 p. 